\title{
The new Handbook Series of Clinical Neurophysiology
}

With pleasure we announce the resumption of the Handbook of Clinical Neurophysiology, first published by Elsevier in 1969. The first series, bound with brown covers, was commissioned at the International Congress in San Diego. Antoine Rémond (IFCN president 1965-1969) served as series editor for the early 1970s volumes. Rémond and Alan Gevins (founder of The San Francisco Brain Research Institute) edited the revised 1980s revised Handbook series, which was blue covered. The brown and blue series focused each volume on a technique. Twenty years ago a new series was commissioned for which Jasper Daube (President of the American Association of Neuromuscular and Electrodiagnostic Medicine 1983-1985) and François Mauguière (IFCN president 2001-2006, Editor-in-Chief of Clinical Neurophysiology 19871995) served as editors. In keeping with a broader scope of the IFCN underway, the term "Electroencephalography" was dropped from the series title. Renamed as the Handbook of Clinical Neurophysiology, this red-bound series focused on clinical fields instead of techniques. Nine volumes appeared between 2003 and 2010: volume 1: movement disorders; volume 2: clinical neurophysiology of disorders of muscle and neuromuscular junction, including fatigue; volume 3: presurgical assessment of the epilepsies with clinical neurophysiology and functional imaging; volume 4: clinical neurophysiology of motor neuron diseases; volume 5: disorders of visual processing; volume 6: clinical neurophysiology of sleep disorders; volume 7: peripheral nerve diseases; volume 8: intraoperative monitoring of neural function; volume 9: vertigo and imbalance: clinical neurophysiology of the vestibular system (https://www.ifcn.info/handbook-series.asp).

The knowledge in the field of clinical neurophysiology is rapidly advancing, driven by the development of new neurotechnological and analytical tools that allow deciphering of pathological changes in the human central and peripheral nervous system with increasing detail and precision. Therefore, time is more than ripe to start a new Handbook series that will update and significantly extend the red series of the Handbook of Clinical Neurophysiology.
One of the previous volume editors (Mark Hallett, Editor of Volume 1 (2003): Movement Disorders) agreed to resume his previous work and will now oversee the updates of this volume in the new Handbook Series, reflecting developments in clinical neurophysiology in the last 10-20 years. However, this volume will only be a start. The IFCN plans to add more volumes to this new IFCN Handbook Series over the next years to cover the full spectrum of diagnostic and therapeutic clinical neurophysiology. It is intended that not only more volumes from the red series of the Handbook will be updated, but also new topics will be covered, such as clinical neurophysiology of neurodegenerative disorders, and clinical neurophysiology of pain.

Every single chapter of a volume of the new IFCN Handbook Series will be published as Invited Review in Clinical Neurophysiology (CLINPH) or Clinical Neurophysiology Practice (CNP). The first chapter of volume 1 (clinical neurophysiology of movement disorders) of the new Handbook Series: Hallett et al. "Evaluation of movement and brain activity" appears in the current issue of CLINPH.

One volume will contain approximately 10 chapters. Each chapter will typically cover a relevant topic and will be written in the form of an up-to-date critical review, typically by globally distributed opinion leaders. In addition to the publication in CLINPH or CNP, all incoming chapters will be continuously collected in an online cross-journal (CLINPH and CNP) Virtual Special Issue (VSI), freely available on ScienceDirect www.sciencedirect.com/ journal/clinical-neurophysiology and www.sciencedirect.com/ journal/clinical-neurophysiology-practice. A VSI can be printed on demand, all or a selection of chapters, with the possible addition of other journal articles. Thus, individually designed books can be created. All chapters of the IFCN Handbook Series will be published gold open access and made freely accessible on the IFCN website (https://www.ifcn.info/). The new Handbook Series of Clinical Neurophysiology will serve as an educational encyclopedia of the IFCN.
UlfZiemann, MD

Editor-in-Cheif, CLINPH
MargittaSeeck, MD

Editor-in-Cheif, CNP 
Ulf Ziemann $^{\mathrm{a}, \mathrm{b}}$

Margitta Seeck ${ }^{c}$

a Department of Neurology \& Stroke, University of Tübingen, Germany

${ }^{\mathrm{b}}$ Hertie Institute for Clinical Brain Research, University of Tübingen, Germany

${ }^{\mathrm{c}}$ Department of Clinical Neurosciences, University Hospitals and Faculty of Medicine of Geneva, Geneva, Switzerland Received 30 May 2021 Accepted 1 June 2021

Available online 19 August 2021 